\title{
A microbiological survey of automated biochemical machines
}

\author{
SWB NEWSOM AND JULIE MATTHEWS \\ From Papworth Hospital, Cambridge, UK
}

SUMMARY The potential microbial hazards to laboratory personnel from operating three pieces of automated laboratory equipment have been tested using challenges of bacteria in serum or water. Aerosols did not seem to be a hazard, and the recommended disinfection procedures seemed quite adequate, although $2 \%$ aqueous activated glutaraldehyde is to be preferred to hypochlorite. The use of plastic gloves for protecting hands when changing rinse cups and $\mathrm{pH}$ electrodes is recommended.

The presence of $\mathrm{HBsAg}$ in a significant number of samples, particularly 'silent' ones from nonjaundiced patients, has caused considerable concern about the potential hazard of using automatic equipment for the chemical analysis of blood. The 'Code of Practice for the prevention of infection in Clinical Laboratories and Post Mortem Rooms'1 notes, among other things, that: 'sample plates must be washed and disinfected daily; at the end of the day the system must be washed with distilled water or the manufacturer's wash fluid; the system must be flushed and disinfected with strong hypochlorite ( $2.5 \%$ chloros) for 10 minutes before changing the dialyser membrane, and disposable gloves must be worn; following 'danger of infection' specimens machines must be washed with $2.5 \%$ chloros or $2 \%$ glutaraldehyde for 10 minutes'.

The loan of an obsolete SMA 6/60 AutoAnalyzer (Technicon), of which four channels were used, and demonstration models of a flame photometer (460Corning) and blood gas analyser (pH 166-Corning) gave us a chance to challenge the machines with tracer microbes to test for any potential hazards and to estimate the effectiveness of the recommended decontamination procedures.

\section{Equipment tested}

The equipment is described from the viewpoint of a microbiologist. The autoanalyser sample tray, the Sampler IV, was a slowly rotating tray containing sample cups, from which serum was aspirated by a hollow probe attached to a suction manifold. After sampling, the probe swung over violently to

Received for publication 21 November 1979 a rinse cup and returned (equally violently) to the next sample. Serum was pumped to four dialyser membranes, each held between plastic blocks. Dialysates were used for the reactions, while the larger molecules from the serum (including any microbes) passed to waste. Thus the reaction side of the machine could only become contaminated if the dialysis membrane leaked. The membrane and associated tubing were changed approximately once a month, as a matter of routine. The flame photometer had a similar rotating sample tray and probe but a different rinse (blank) cup and an electrolyte standard cup. The rinse (blank) cup was a small sealed plastic cylinder, which was impaled on a needle which fed it with rinse (blank) fluid. The sampler probe then entered the cylinder through the 'roof' after perforating the plastic. At regular intervals the sampler probe passed through gaps in the sample tray and penetrated a second plastic cylinder sited below, this time flushed with the standard solution. Both rinse and standard cylinders had to be changed daily. Serum samples were dialysed, and the dialysates were diluted and run into a nebuliser feeding the flame; any excess joined the serum waste in a catch pot before being passed into the drain. The instrument had a 10-minute automatic flush period before use.

The gas analyser was charged with blood from a syringe inserted into a female connector at the front. As the blood was injected a valve above a vent tube at the side of the machine opened to relieve the pressure. The blood passed into the electrode chamber and after the measurement a vacuum pump sucked a flushing fluid through the system. Excess blood and rinse fluid passed into the vent tube and then into a waste bottle fitted with an automatic 
cutout to prevent overflowing; between the bottle and the vacuum pump was a tube containing a protein trap, in this case a cotton-wool filter, although on newer models a disposable cellulose fibre cartridge is used. The machine was calibrated by standard gases which were humidified by passing through two tubes on the left side; any excess gas was bled through a valve sited on the front next to the entry port.

\section{Material and methods}

Bacillus subtilis var globigii (NCTCC No. 10073) spores, and Escherichia coli (NCTCC No. 104188) cells were used as tracers in either serum or water at $10^{8}$ per ml. Cultures were made on nutrient agar for $B$. subtilis and on MacConkey agar for $E$. coli. The cultures were incubated for three days at $37^{\circ} \mathrm{C}$. Numbers of bacteria were estimated by the Miles and Misra dropping method, or by filtration through a $0.22 \mu$ membrane filter; rough counts were obtained by plating a single loopful or a swab. For some tests, Brain Heart Infusion (Difco) was used. A Cassella slit sampler set to operate at $271 / \mathrm{m}$ was used to sample for aerosols. The disinfectants used were $2 \%$ activated glutaraldehyde (Cidex, Asep, 3M), 2\% plain glutaraldehyde, hypochlorite ( $2.5 \%$ Chloros), and $2 \%$ activated glutaraldehyde with detergent (Clinicide). Disinfectant test pieces were made as for ethylene oxide steriliser tests ${ }^{2}$ with $10^{6} \mathrm{~B}$. subtilis spores dried from water on to aluminium foil pieces. The number of bacteria per piece was estimated after resuspending some in peptone with a sonic vibrator to distribute the spores evenly. A more stringent test was provided by covering some of the pieces with serum. The pieces were immersed in the disinfectant under test, and sets of five were removed at intervals from 15 minutes to 24 hours later. The residual glutaraldehyde was neutralised by dipping the pieces in $1 \%$ sodium bisulphite (which did not affect the viability of the spores), and then the pieces were tested for sterility by incubation in Brain Heart Infusion broth for five days at $37^{\circ} \mathrm{C}$. Any growth was checked to ensure it was $B$. subtilis. The tests and results are recorded together for clarity. The autoanalyser and flame photometer were sufficiently alike to warrant similar tests, but the blood gas analyser required a different approach.

\section{Tests and results}

AUTOANALYSER AND FLAME PHOTOMETER The following aspects were tested: sampling, rinsing, the dialysis membrane holders, the nebuliser and catch pot (flame photometer), serum and reagent effluents, disinfection, and flushing.

\section{Sampling}

Sample cups were filled with the four different challenges on separate occasions, and the machines were run. Twelve tests of the air using the slit sampler beside the autoanalyser, and six beside the flame photometer, failed to reveal any aerosols. Twenty-three plates exposed on horizontal autoanalyser surfaces and six on the flame photometer revealed only four and two splashes respectively. However, sterile blotting paper exposed on vertical surfaces and then cultured revealed heavy contamination.

\section{Rinsing}

After a run the rinse fluids of both machines contained approx. $10^{3}$ microbes $/ \mathrm{ml}$. The flame photometer had a 10-minute rinse before use, and this reduced the spore count by 100 -fold but apparently did little to that of $E$. coli. The flame photometer standard cup was less contaminated, and, after flushing, all the bacteria had disappeared from it.

\section{Dialyser membrane blocks}

The surfaces of the plastic blocks holding the membranes were swabbed to see if any microbes leaked out during the run or after a membrane change on a contaminated system. When the blocks were separated to remove the membrane, their inner surfaces were swabbed. The sides and fronts of the blocks on both machines were free from contamination; the inner 'serum-side' of all blocks was heavily contaminated (as expected) but the 'reagent sides' were sterile (or free of tracer microbes). This clearly demonstrates the value of the membranes as antibacterial filters protecting the rest of the systems. After changing the membranes both the sides and fronts of the blocks were contaminated and so required to be cleaned.

\section{Tubing connectors and rinse cups}

Sterile plastic gloves were used when changing the tubing and also when removing the flame photometer rinse and standard cups. The gloved fingers were then impressed on the surface of an agar plate. The surfaces around a newly made junction were then swabbed. All these tests were made after a run with contaminated sera and without decontamination. Six autoanalyser connections were changed, altogether on nine occasions. The fingers were lightly contaminated in four of the nine, and bacteria were recovered from the outside of three junctions. Similar findings applied to the flame photometer. The flame photometer rinse and standard cups were changed after flushing the machine for 20 minutes and then leaving overnight. 
The fingers were moderately contaminated by this procedure.

\section{Nebuliser and catch pot (flame photometer)}

Fourteen samples of the air beside the catch pot (which appeared 'bubbly') and 10 from above the nebuliser (used without the flame) failed to reveal aerosols.

\section{Serum and reagent effluent}

In both machines the serum waste was highly contaminated after a run; flushing with water for 5 minutes reduced the count slightly in the autoanalyser, and the 10-minute automatic flush in the photometer reduced the bacteria in the effluent to an undetectable level (despite filtering $50 \mathrm{ml}$ ). The reagent wastes of both machines were usually sterile, but the autoanalyser drain occasionally had counts of up to 25 spores per $50 \mathrm{ml}$ of effluent (which compares with the original challenge of $10^{8} / \mathrm{ml}$ ). Even samples taken after a membrane change were sterile.

\section{Disinfection}

The $2 \%$ activated and plain glutaraldehydes were used on the autoanalyser together with the hypochlorite, while the $2 \%$ glutaraldehyde with detergent was used on the flame photometer. The reagents were flushed through the autoanalyser tubes for 5 minutes (including reagent tubes). The effluents were then cultured. The $2 \%$ activated glutaraldehyde gave the best results in the autoanalyser, hypochlorite was almost as good, and the non-activated glutaraldehyde worked well on $E$. coli but had no effect on spores. The disinfection of the flame photometer was impossible to assess as the flushing had removed all the bacteria.

\section{BLOOD GAS ANALYSER}

Challenges of $B$. subtilis-loaded blood were injected into the machine, flushed, and repeated four times. Air was sampled at the front, and plates were exposed below the entry port to catch any droplets. The air expelled from the vent tube during the process was bubbled through some Brain Heart Infusion broth, as was the vacuum pump exhaust air. The electrodes were then removed and swabbed, and the cotton wool 'protein trap' protecting the vacuum pump was removed and cultured. No detectable aerosols were generated by injecting blood, but the model tested had a fault that allowed a drop to fall back out of the machine and contaminate the bench below; wrong adjustment of the flush solution drip rate may have a similar effect. The leak on the model tested was due to a faulty fluidic diode, which is readily rectifiable by the service engineer. Air from the vent tube yielded some $B$. subtilis (although not counted), and the electrodes were (naturally) heavily contaminated; however, the protein trap and the vacuum pump air were sterile. The liquid in the calibration gas humidifiers was heavily contaminated with Gram-positive cocci and Gram-negative rods, and some of the cocci were pushed into the air through the front vent valve during calibration.

\section{DISINFECTANT TESTS}

The results of the disinfectant tests using the five types of glutaraldehyde are seen in the Table. The glutaraldehyde-detergent mixture worked the most rapidly and so is to be preferred.

\section{Effectiveness of different glutaraldehydes}

\begin{tabular}{lllllll}
\hline & & \multicolumn{3}{c}{ Exposure time (min) } \\
\cline { 3 - 6 } & & & 15 & 30 & 60 & $I 20$ \\
\hline $2 \%$ activated (Clinicide) & Serum & 2 & 2 & 1 & 0 \\
& Water & 0 & 0 & 0 & 0 \\
$2 \%$ activated (Cidex) & Serum & 5 & 5 & 4 & 3 \\
& Water & 4 & 4 & 0 & 1 \\
& Serum & 5 & 5 & 5 & 5 \\
$2 \%$ not activated & Water & 5 & 5 & 5 & 5 \\
& Serum & 5 & 5 & 1 & 0 \\
& Water & 1 & 0 & 0 & 0 \\
& Serum & 5 & 4 & 0 & 0 \\
& Wativated (Asep) & 3 & 0 & 0 & 0 \\
\hline
\end{tabular}

Numbers $=$ No. of test pieces (out of 5) growing spores after exp.sure for the stated time.

\section{Discussion}

Our tests showed that the samplers created less of a hazard than might have been suspected. Only the vertical surfaces around the samplers seemed really at risk. The results suggest that the design of any machine including a sampler should allow for smooth, crack-free, and easily cleanable surfaces nearby; and that a system in which the probe is not swung violently from side to side would be an advantage.

The rinse cups, or any other standard cups, must become contaminated and should be regarded as such when being handled. The lack of an aerosol may seem surprising, although the MRC Working Party on Haemodialysis ${ }^{3}$ found it hard to generate an aerosol from blood, while Rutter and Evans ${ }^{4}$ failed to detect one from an autoanalyser stirrer. The challenges of bacteria in water would have been a better source of aerosols, but even these did not create a hazard. The dialysis membranes (while competent) obviously acted as good antibacterial filters, so that most of the flow pathways in the machines were free of contamination. Presumably a leaky membrane would fail from a biochemical 
viewpoint and so become readily detectable. Provided the membrane was fitted properly no bacteria leaked out on to the surfaces of the holding blocks, although the latter became contaminated after a nondecontaminated membrane was changed.

The serum pathways of both machines were naturally heavily contaminated, but the flushing mechanism of the flame photometer was very effective at clearing it. Flushing the autoanalyser was less effective, presumably because of the more complex pathway. Thus, although to change reaction tubing would not present much hazard, to change the serum tubing would. The suggested disinfection worked satisfactorily. Activated glutaraldehyde was the best agent, which is explicable in that chlorine is neutralised by protein. The biochemists prefer glutaraldehyde because it does not react with the metal connectors found in some machines. Nonactivated glutaraldehyde worked well on $E$. coli but not on spores, and would not be recommended; the glutaraldehyde with detergent is to be preferred as it had the quickest killing action.

The blood gas analyser was a simpler piece of equipment, and although some spores leaked out of the vent tube, these are unlikely to represent a hazard with respect to hepatitis, which rarely if ever is spread by aerosols. The WHO monograph ${ }^{5}$ regarded the parenteral route of infection as the most likely, and their conclusions are borne out by the observation that far more laboratory-acquired hepatitis infections can be accounted for by accidents than can other laboratory-acquired infections. For example, an epidemiological study of a laboratory epidemic $^{6}$ using risk factor analysis and matching the five affected employees for age, length of employment, and degree of exposure to blood with healthy controls, showed that the only significant factor was a history of sustaining cuts on the fingers while handling sharp-edged computer request cards; and, indeed, one of the five spilt blood from an infected patient over his hands.

Care was obviously required in handling the electrodes, and plastic gloves would be needed to protect the hands. More disturbing is the finding of Gram-negative rods in the humidifier tubes, with a possibility of their escape from the apparatus through the front vent tube. Pseudomonas aeruginosa is very often found in stagnant humidifier water, and indeed was found in our own blood gas analyser sited inside the special care baby unit (although of a serotype unrelated to that found in the babies). Nevertheless, the use of such apparatus in high-risk clinical areas within the hospital must be viewed with some suspicion. The back flow of blood from this unit represents a functional defect and is not normally found. If this were a frequent fault, then benches could become contaminated by blood and may also do so with rinse buffer if the supply valve is opened too far. Hughes and $\mathrm{Cox}^{7}$ found Pseudomonas cepacia in the tonometer waterbath and rinse bottles of the blood gas equipment located in their intensive care ward and noted that unopened bottles of the rinse buffer (Instrumentation Laboratories Inc) contained the same organism. They were not able to trace any infections due to this organism but felt that users should be warned that the buffers were not necessarily sterile.

Our studies showed that the hazards of automatic apparatus for biochemistry are not as great as may be imagined, a finding that is in accord with Professor Grist's observation ${ }^{8}$ that laboratory-acquired hepatitis was no more common in laboratories with automated equipment than in those without.

The instructions for sterilisation in the 'Code of Practice' seem adequate with regard to routine cleaning (section 25a) with the exception of the failure to emphasise the dangers of surfaces around the sampler as well as the sample plate, and the dangers of rinse cups. Activated glutaraldehyde would seem to be a better and safer (if more expensive) disinfectant than hypochlorite but is not mentioned in this section, although it appears in section $25 \mathrm{~b}$ as an alternative to hypochlorite for danger of infection specimens. This distinction seems unnecessary; and anyway to some extent all specimens are potentially hazardous, and the machines should always be regarded as contaminated.

We are grateful to the Department of Biochemistry, Addenbrooke's Hospital, and to Corning Ltd for the loan of apparatus. This work was supported by the Inter-Authority Engineering Study Group 9, the Engineering Division of the Department of Health and Social Security. We are also grateful to Mr DA Kennedy, The Scientific and Technical Branch of the DHSS, for helpful suggestions.

\section{References}

${ }^{1}$ Howie Sir JW, chairman. A code of practice for the prevention of infection in Clinical Laboratories and Post-Mortem Rooms (DHSS, Working Party). London: HMSO, 1978.

${ }^{2}$ Beeby MM, Whitehouse CE. A bacterial spore test piece for the control of ethylene oxide sterilisation. $J$ Appl Bacteriol 1965;28:349-60.

${ }^{3}$ MRC Working Party on Haemodialysis. Experimental studies on environmental contamination with infected blood during haemodialysis. J Hyg (Cambr) 1975;74:133-48.

${ }^{4}$ Rutter DA, Evans CGT. Aerosol hazards from some 
clinical laboratory apparatus. Brit Med J 1972;i: 594-7.

${ }^{5}$ World Health Organisation. Model code of laboratory practice for dealing with Hbag. Geneva, 1973.

${ }^{6}$ Pattison CP, Boyer KM, Maynard JE, Kelly PC. Epidemic hepatitis in a clinical laboratory: possible association with computer card handling. JAMA 1974;230:854-7.

${ }^{7}$ Hughes WT, Cox F. Are blood gas machines safe for use in the intensive care nursery? Paediatrics 1977; 60:760-1. (Letter.)

${ }^{8}$ Grist NR. Hepatitis in clinical laboratories. J Clin Pathol 1975;28:255-9.

Requests for reprints to: Dr SWB Newsom, Sims Woodhead Memorial Laboratory, Papworth Hospital, Papworth Everard, Cambridge CB3 8RE, UK. 